\title{
Asthma survey items as predictors of respiratory problems in children 2 yrs later: a longitudinal study
}

\author{
P.K. Pattemore*, F.C. Lampe**, S. Smith ${ }^{+}$, J.B. Clough ${ }^{\ddagger}$, S.T. Holgate ${ }^{+}$, S.L. Johnston ${ }^{+}$
}

\begin{abstract}
Asthma survey items as predictors of respiratory problems in children 2 yrs later: a longitudinal study. P.K. Pattemore, F.C. Lampe, S. Smith, J.B. Clough, S.T Holgate, S.L Johnston. C ERS Journals Ltd 1999.

ABSTRACT: The study compared the ability of characteristics defined by an asthma survey (wheeze versus cough and asthma diagnosis versus no diagnosis) to predict later respiratory problems in a cohort of 108 schoolchildren who had reported either recent wheeze or recurrent cough in a 1987 asthma survey.

The children recorded daily respiratory symptoms and peak flow from April 1989 until May 1990. The frequency and severity of lower respiratory symptom episodes and peak flow dips were compared in the wheeze and cough groups and in the diagnosed versus nondiagnosed children. The independent effects of initial wheeze, atopy, diagnosis and bronchial hyperresponsiveness (BHR) on the longitudinal outcome measures were assessed using multiple linear regression.

Children with initial wheeze had more chronic symptoms and peak flow variability than those with cough alone, but wheeze had only a weak effect on frequency and severity of acute lower respiratory episodes. Children with both wheeze and atopy had more acute symptomatic episodes and more chronic symptoms than did the other children. Children with diagnosed asthma (versus no diagnosis) had significantly more frequent and severe lower respiratory exacerbations, more days symptomatic and greater peak flow variability. The predictive effects of diagnosis were independent of (and stronger than the effects of) wheeze, atopy and BHR, or combinations of these variables.
\end{abstract}

The results suggest that among children who report respiratory symptoms, surveyreported wheeze on its own is a weaker marker of significant respiratory disease than is a doctor's diagnosis of asthma.

Eur Respir J 1999; 14: 650-658.

Surveys of childhood asthma such as the International Study of Asthma and Allergies in Childhood (ISAAC) [1] have provided important information about worldwide geographical differences in prevalence and severity of asthma and other atopic disorders [2]. Such surveys record and analyse symptoms of wheeze and nocturnal cough, and a diagnosis of asthma as indices of asthma. In some studies, skin testing or bronchial challenge are also performed. Of the questionnaire items, wheeze is regarded as a more specific marker of asthma than recurrent cough, and is often used as the primary index of asthma prevalence. Asthma diagnosis is felt to be a locally variable entity and an underdiagnosis is common [3]. Few studies to date have examined how closely these surveyderived categories predict respiratory problems over time $[4,5]$ although numerous cross-sectional studies have compared reported symptoms with bronchial hyperresponsiveness (BHR) [6].

This study presented a unique opportunity to examine this issue using longitudinal daily symptom and peak flow data in a cohort of Southampton school children who were selected in 1987 on the basis of asthma questionnaire responses and atopic status. The cohort was followed in the authors' unit until 1990. Acute and chronic respiratory
*Dept of Paediatrics, Christchurch School of Medicine, University of Otago, Christchurch, New Zealand. **Dept of Primary Care and Population Sciences, Royal Free Hospital School of Medicine, University of London, London, UK. 'University Medicine, University of Southampton, Southampton, UK. "Dept of Child Health, University of Southampton, Southampton, UK.

Correspondence: P.K. Pattemore, Dept of Paediatrics, Christchurch School of Medicine, P.O. Box 4345 Christchurch, New Zealand. Fax 06433640919

Keywords: Asthma, children, cough, diagnosis, respiratory acerbations, wheeze

Received: September 101998

Accepted after revision April 121999

The study was supported by grants from Action Research, the National Asthma Campaign, the British Lung Foundation; the British Medical Association, the Chest, Heart and Stroke Association, and Miles Inc., New Haven, Connecticut. Allen \& Hanbury's UK Ltd and Clement-Clarke Ltd supplied peak flow meters for the study. The Medical Research Council of New Zealand, the Asthma Foundation of New Zealand, and Allen \& Hanbury's New Zealand Ltd supported P.K. Pattemore. problems derived from the 1989-1990 longitudinal data recordings could be analysed according to characteristics available at initial selection in 1987.

The aim of the present study was to determine whether or not children reporting wheeze in a questionnaire survey had more acute or chronic respiratory problems than children reporting cough without wheeze. A second aim was to compare common survey-derived indices of asthma prevalence, particularly wheeze and asthma diagnosis, as predictors of later respiratory illness.

\section{Materials and methods}

The methods of selection and follow-up of the cohort have been previously described $[7,8]$, and are summarized briefly.

\section{Study subjects}

Initial selection in 1987. In 1987, Clough et al. [4, 9] surveyed 3,698 children aged 7-8 yrs in the Southampton region for asthma symptoms. They selected a random subsample of those children who reported asthma symptoms in that survey and skin-tested them with a panel of common allergens (house dust mite, i.e. Der pl, mixed 
grass pollens, cat allergen; Bencard, Brentford, Middlesex, UK), and histamine and saline. They defined atopy as at least one skin test positive ( $\geq 2 \mathrm{~mm}$ wheal diameter). They then randomly selected 48 children, using a quota filling system, from within each of the following four groups: 1) atopic children who reported wheeze (with or without cough) in the previous year; 2) atopic children who reported recurrent cough without wheeze in the previous year; 3) nonatopic children reporting wheeze; 4) nonatopic children reporting recurrent cough without wheeze.

Of the 192 children so selected, CLOUGH et al. [4, 9] followed 183 for 12 months (1987-1988) with daily symptom and twice daily peak flow recordings.

Ascertainment of asthma diagnosis and bronchial hyperresponsiveness in 1987. At recruitment to the 1987 study Clough et al. [4, 9] had examined the primary practitioner's notes of all children to ascertain if the child had a diagnosis of asthma. They had also measured the BHR to methacholine using the method of YAN et al. [10]. (BHR defined as the provocative dose of methacholine causing a $20 \%$ fall in forced expiratory volume in one second $(\mathrm{PD} 20) \leq 6.4 \mu \mathrm{mol})$.

Participation and informed consent in the current stu$d y$. These same children and their families were invited to participate in a further follow-up period from 3 April 1989 to 7 May 1990 to identify and study acute respiratory episodes, including taking nasal aspirates and blood tests for viruses (the results of the virus testing have been reported elsewhere [7, 8]. The families of 114 children agreed to the current study, of which 108 successfully completed the study.

The Southampton Hospitals Joint Ethical Committee approved the study, and informed consent was obtained from parents of all the children.

\section{Study design}

This was a prospective longitudinal cohort study which was designed to identify and study acute episodes of respiratory symptoms and peak flow variations over $1 \mathrm{yr}$ of follow-up. Daily symptoms and peak flow were recorded for 13 months, and were analysed by computer for acute episodes of deterioration, chronic symptoms and peak flow variability. Summary measures of acute and chronic respiratory problems between 1989 and 1990 were used as outcome measures. Multiple linear regression was used to model the independent effects of predictive variables obtained at the time of recruitment in 1987.

\section{Methods}

Symptom and peak flow recording. Each morning and evening of the study, children recorded the best of three readings of peak expiratory flow (PEF), performed on mini-Wright peak flow meters (Clement Clarke International, Harlow, Essex, UK). Each evening they completed symptom records. Symptoms present (cough by day and by night, wheeze by day and by night, difficulty breathing, and inability to attend school because of chest problems) were scored on a scale of 0 (absent) to 3 (severe) and the scores were added each day to give a total lower respiratory tract (LRT) score.
Analysis

Definitions. The group of children selected for wheeze (with or without cough) were referred to as the "children selected for wheeze", and the group of children selected for recurrent cough without wheeze, as the "children selected for cough".

The word "episode" was used to indicate a respiratory event detected by computer analysis of the children's daily records over the entire study. These episodes have been defined previously [11], but are described here, as they are key terms in this analysis. Two physicians independently went through the plotted time charts of symptoms and $\mathrm{PEF}$ and decided what constituted a significant episode, the start date and end date of each episode, and what differentiated two consecutive episodes from one long episode. Computer algorithms of LRT and of PEF episodes that best fitted these physicians' independent assessments were chosen as follows:

A significant "episode" of LRT symptoms was defined as a cluster of at least 2 days when total LRT symptom scores were above that child's median symptom score for the year, provided that scores were at or below the median on the day before and 2 days after the cluster. The duration of a symptom episode was defined as the number of days the score was above the median value. The severity was defined as the peak symptom score during the episode. Figure 1a shows an example of a symptom episode.

Peak flow values had been adjusted for growth through the year. A significant PEF "episode" was defined as a cluster of at least 2 days when PEF was at or below the child's 10th percentile for morning PEF recordings for the year. In addition, the definition required that the PEF was above the 10th percentile on the day before the cluster, and at or above the median PEF for at least 2 days after the cluster. The duration of a PEF episode was defined as the number of inclusive days from the first day PEF was below the 10th percentile to the day it was below the median. The severity was defined as the greatest deviation from the median during the episode. Figure $1 \mathrm{~b}$ shows an example of a PEF episode.

In this way, each child's episodes were identified against that child's own background level of reporting, which functioned as an internal control. The LRT and PEF episodes were identified independently of each other by computer analysis. Thus, a single illness might qualify either as an LRT episode, or as a PEF episode, or as both.

Outcome measures. Summary episode measures calculated for each child were: the annual rates of PEF and LRT episodes (using each child's nonmissing follow-up time as the denominator), the mean severity of PEF and of LRT episodes and the mean duration of PEF and of LRT episodes. For the purposes of group comparisons, six children who had no LRT episodes were given a mean severity score of zero. In addition to acute episodes, the following two indices of chronic illness were also defined for each child: percentage of days on which any LRT symptoms were recorded, and coefficient of variation of morning PEF (the standard deviation as a percentage of the mean for the whole study period).

Predictive variables. The predictive variables were all derived from the time of entry in 1987 from the study 

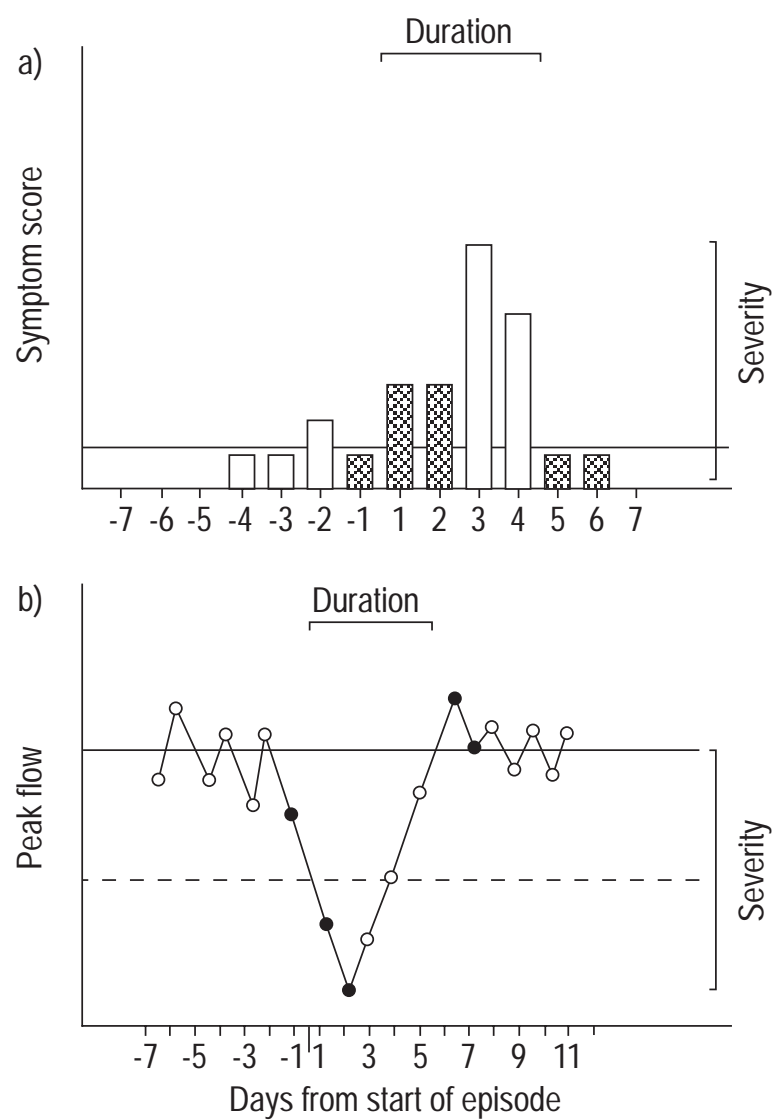

Fig. 1. - Hypothetical examples of a) a lower respiratory tract (LRT) symptom episode and b) a peak expiratory flow (PEF) episode, indicating the parameters defined in the Materials and methods section. : the minimum set of data points required to meet the definition of an episode (in relation to the child's median symptom score or PEF $(-)$ and the 10th percentile PEF (- - - ) for the year. The duration of a symptom episode was defined as the number of days the score was above the median value, while the severity was defined as the peak symptom score during the episode. The duration of a PEF episode was defined as the number of days from the day PEF was below the 10th percentile to the day it was below the median, while the severity was defined as the greatest deviation from the median during the episode. $\square$ and $\bigcirc$ represent any other points occurring in an episode in addition to defined points.

of Clough et al. [4, 9] as follows: 1) wheeze in the previous 12 months reported in the initial parentcompleted questionnaire [4, 9]. The comparison groups were those who had reported recurrent cough but no wheeze; 2) atopy defined by skin tests as above; 3 ) a previous doctor's diagnosis of asthma; and 4) BHR.

\section{Statistical analysis}

All outcome variables were log 10 transformed prior to analysis in order to normalize distributions and stabilize variance. For variables that had zero values, 1.0 was added prior to taking logs. In addition, t-tests were used to assess the effect of diagnostic status on log transformed outcome variables. Multiple linear regression was undertaken to assess the effects on logged outcome variables of: 1) wheeze compared to cough after adjustment for atopic status (because the selection of wheeze and cough groups had been stratified by atopic status); 2) the interaction between wheeze and atopy; 3 ) the independent effects of predominant symptom, atopic status, diagnosis status, and BHR status (all measured at the time the children were selected in 1987); and 4) the effect of combinations of these factors.

Regression coefficients were anti-logged and can be interpreted as ratios of geometric means which are presented with 95\% confidence intervals (CI). Proportions were compared using the Chi-squared statistic (without continuity correction).

\section{Results}

\section{Subject characteristics}

Of the 183 children who completed the 1987-1988 study, 75 elected not to participate in the current study. The most common reason for nonparticipation was the more invasive nature of nasal aspirates and blood tests in the current study. The distributions by age, sex, predominant symptom, atopic status, asthma diagnosis and BHR did not differ significantly between participants and non-participants (table 1: a further three children who commenced but did not complete the 1987-1988 study [4, 9] are included among the nonparticipants).

At the start of the present study, the mean age of the children in the cohort was 9.6 yrs. (range $8.7-10.7 \mathrm{yrs}$ ) and $58(54 \%)$ of the children were male. A breakdown of the cohort by asthma-related categories is given in table 2 . The 108 participants were still distributed fairly equally among each of the four subgroups within which they had been randomly selected in 1987 by CLOUGH et al. [4, 9]. Fifty-two $(48 \%)$ children had been initially selected for reporting wheeze, and $42(39 \%)$ in total had a previous diagnosis of asthma.

The likelihood of having an existing diagnosis of asthma was greater in children initially selected for wheeze rather than cough (58 versus $\left.21 \%, \chi_{1}^{2}=14.92, p<0.001\right)$ and in atopic rather than nonatopic children (53 versus $26 \%, \chi_{1}^{2}=$ $7.73, \mathrm{p}=0.005)$. It was especially high among atopic children selected for wheeze compared to nonatopic children selected for cough (76 versus $11 \%$ ). Similarly BHR was more common among children with wheeze than among those with cough (58 versus $\left.23 \%, \chi_{1}{ }^{2}=13.38, p<0.001\right)$, among those atopic than among those nonatopic (47 versus $34 \%, \chi_{1}{ }^{2}=1.92, \mathrm{p}=0.166$ ), and particularly among those selected for wheeze and atopy than among those selected for cough and no atopy (72 versus $22 \%$ ).

Fifty-three (49\%) children had at least one identifiable episode where wheeze was reported during the follow up period of the present study, including 37 of the 52 initially selected for wheeze $(71 \%)$, and 16 of the 56 initially selected for cough without wheeze $(29 \%)$.

\section{Computer-defined episodes of lower respiratory problems}

Computer analysis identified a large number of episodes. For the 108 children, the median (range) of each LRT episode summary measure was $3.7(0-25)$ for the annual episode rate, $3(1-11)$ for mean episode severity and 6 days (3-27 days) for mean episode duration. For PEF episodes, the median (range) was $4.2(0.9-8.4)$ for the annual episode rate, $55 \mathrm{~L} \cdot \mathrm{min}^{-1}\left(12-176 \mathrm{~L} \cdot \mathrm{min}^{-1}\right)$ for mean 
Table 1. - Comparison of participants and nonparticipants in the 1989-1990 study

\begin{tabular}{lcccr}
\hline Characteristic & $\begin{array}{c}\text { Total 1987 study } \\
(\mathrm{n}=186)\end{array}$ & $\begin{array}{c}\text { Current participants } \\
(\mathrm{n}=108)\end{array}$ & $\begin{array}{c}\text { Nonparticipants } \\
(\mathrm{n}=78)\end{array}$ & 95\% CI on differences $^{+}$ \\
\hline Age yrs* & $9.59 \pm 0.58$ & $9.56 \pm 0.57$ & $9.64 \pm 0.59$ & $-0.09-0.25$ \\
& $(8.68-10.66)$ & $(8.71-10.66)$ & $(8.68-10.63)$ & $-6.5-22.2$ \\
Male \% & 57.0 & 53.7 & 61.5 & $-10.1-19.0$ \\
Wheeze \% & 50.0 & 48.2 & 52.6 & $-13.3-15.9$ \\
Atopic \% & 50.5 & 50 & 51.3 & $-8.4-20.4$ \\
Asthma diagnosis \% & 41.4 & 38.9 & 44.9 & $-10.6-18.1$ \\
BHR \% & 41.4 & 39.8 & 43.6 & \\
\hline
\end{tabular}

*: Age on 3 April 1989, data presented as meanplusmn;SD (range); ${ }^{+}$: current participants versus nonparticipants. CI: confidence interval; BHR: bronchial hyperresponsiveness.

episode severity (fall in PEF), and 16 days (3-84 days) for mean episode duration. The longer duration of PEF episodes may reflect the way PEF episodes were defined [8] or lagging of lung function resolution behind symptom resolution. There were no sex differences in any of these categories.

\section{Effect of wheeze versus cough alone}

As the original selection of children with wheeze and cough was stratified by atopic status, comparisons between wheeze and cough were adjusted for atopic status. After adjustment, children selected for wheeze had increased chronic respiratory morbidity compared to those with cough alone (table 3 ). The wheeze group had significantly greater percentage of symptomatic days (adjusted ratio of geometric mean (GM) 1.51) and coefficient of variation of PEF (adjusted ratio of GM 1.27). Wheeze had a lesser effect on acute episode rate and severity: the LRT episode severity was significantly increased in children with wheeze compared to cough (adjusted ratio of GM 1.23) and the LRT episode rate showed a moderate but nonsignificant increase. These differences were very similar to those in unadjusted comparisons between the wheeze group and the cough only group.

\section{Interaction between wheeze and atopy}

Figure 2 shows median episode rates and severity, per cent of days with LRT symptoms and PEF coefficient of variation, in the four groups defined by combination of

Table 2. - Breakdown of the study cohort by four asthmarelated categories

\begin{tabular}{|c|c|c|c|c|c|c|}
\hline & \multicolumn{2}{|c|}{ Wheeze } & \multirow[b]{2}{*}{$\begin{array}{l}\text { Block } \\
\text { total }^{\#}\end{array}$} & \multicolumn{2}{|c|}{ Cough } & \multirow[b]{2}{*}{$\begin{array}{l}\text { Block } \\
\text { total }^{\#}\end{array}$} \\
\hline & BHR & $\begin{array}{c}\text { No } \\
\text { BHR }\end{array}$ & & BHR & $\begin{array}{c}\text { No } \\
\text { BHR }\end{array}$ & \\
\hline Atopy & & & 25 & & & 28 \\
\hline Diagnosis & 16 & 3 & & 4 & 5 & \\
\hline No diagnosis & 2 & 4 & & 3 & 16 & \\
\hline No atopy & & & 26 & & & 27 \\
\hline Diagnosis & 8 & 3 & & 1 & 2 & \\
\hline No diagnosis & 4 & 11 & & 5 & 19 & \\
\hline Missing atopy data & & & & & & \\
\hline No diagnosis & & 1 & & & 1 & \\
\hline
\end{tabular}

Forty-eight children were originally recruited from each of the four blocks defined by symptom and atopic status. ${ }^{\#}$ : the number of children in these blocks in the current study. Equal representation in the present study would imply 27 children in each block. BHR: bronchial hyperresponsiveness. the predominant symptom and atopic status. Tests for an interaction between symptom and atopic status (to test whether the effect of the symptom was different among children with atopy than those nonatopic children) were not significant for any outcome variable, but this may have been affected by study power. Compared to the other three groups, the children selected for both wheeze and atopy had significantly higher values for the following (ratio of GM; 95\% CI): annual LRT episode rate (1.59; $1.15-2.19)$, mean PEF episode severity $(1.27 ; 1.00-1.62)$, percentage of days with LRT symptoms $(2.04 ; 1.30-$ $3.22)$ and coefficient of variation of PEF $(1.28 ; 1.01-$ $1.63)$.

\section{Effect of pre-existing diagnosis}

A pre-existing primary practitioner diagnosis of asthma was highly predictive of respiratory morbidity. Compared to undiagnosed children, children with a diagnosis had considerably increased rate of LRT episodes (ratio of GM; $95 \%$ CI) $(1.89$; 1.46-2.44), mean severity of LRT episodes $(1.33 ; 1.10-1.60)$, mean severity of PEF episodes (1.29; $1.05-1.59)$, percentage of symptomatic days $(2.50 ; 1.73-$ $3.61)$ and coefficient of variation of PEF (1.50; 1.23-1.82). The PEF episode rate was not significantly increased in children with a diagnosis compared to those without (1.13; 0.93-1.38). All of these associations were stronger than for the combination of wheeze and atopy.

\section{Independent effects of classification variables}

The strong predictive effects of diagnosis might only reflect the high proportion of wheeze, atopy and BHR in the diagnosed group, factors that are more commonly used to measure asthma prevalence. As a result the predictive effects of a diagnosis with adjustment for all of these factors was assessed. Table 4 presents the adjusted effects of each classification factor (present versus not present) on each outcome variable. After adjustment for other factors, the effects of wheeze and atopy were greatly attenuated and neither factor remained independently associated with acute or chronic outcome measures. Adjustment for other factors only slightly attenuated the effect of a diagnosis on all outcome variables: diagnosed asthma remained independently associated with the LRT episode rate and severity, the percentage of symptomatic days, and the peak flow variability. HR caused a marked increase in peak flow variability which was independent of other factors, and also tended to increase the LRT episode 
Table 3. - Regression models with adjustment for wheeze and atopy. Analysis of log transformed data

\begin{tabular}{|c|c|c|c|c|}
\hline \multirow[b]{3}{*}{ Dependent variable } & \multicolumn{4}{|c|}{ Independent variables } \\
\hline & \multicolumn{2}{|c|}{ Wheeze effect } & \multicolumn{2}{|c|}{ Atopy effect } \\
\hline & $\begin{array}{l}\text { Adjusted ratio of means } \\
\qquad(95 \% \mathrm{CI})\end{array}$ & p-value & $\begin{array}{l}\text { Adjusted ratio of means } \\
\qquad(95 \% \mathrm{CI})\end{array}$ & p-value \\
\hline \multicolumn{5}{|l|}{ Annual rates } \\
\hline LRT episodes & $1.28(0.97-1.69)$ & 0.079 & $1.26(0.96-1.67)$ & 0.095 \\
\hline PEF episodes & $1.02(0.84-1.24)$ & 0.861 & $1.05(0.87-1.29)$ & 0.567 \\
\hline \multicolumn{5}{|l|}{ Mean severity } \\
\hline LRT episodes & $1.23(1.02-1.49)$ & 0.031 & $1.13(0.93-1.36)$ & 0.215 \\
\hline PEF episodes & $1.15(0.93-1.41)$ & 0.193 & $1.01(0.82-1.25)$ & 0.898 \\
\hline \multicolumn{5}{|l|}{ Chronicity } \\
\hline Days symptomatic \% & $1.51(1.02-2.23)$ & 0.039 & $1.42(0.96-2.10)$ & 0.077 \\
\hline PEF coefficient of variation $\%$ & $1.27(1.04-1.56)$ & 0.022 & $0.96(0.78-1.17)$ & 0.669 \\
\hline
\end{tabular}

All values are ratios of geometric means (95\% confidence intervals (CI)), adjusted using both wheeze and atopy in the model. LRT: lower respiratory tract; PEF: peak expiratory flow.

rate and PEF episode severity, although these effects were not significant.

Two further binary variables, the combination of both wheeze and atopy (compared to either or neither) and the combination of both wheeze and BHR, were examined as predictors of the outcome variables. Both variables have been used in various asthma surveys as surrogates of important clinical asthma. Each variable was included in turn as independent variables alongside a diagnosis of asthma in regression models (table 5).

In the first set of regressions, including "wheeze + atopy" and diagnosis, an asthma diagnosis remained a strong independent predictor of LRT episode rate and severity, percentage of symptomatic days and PEF variability, and again had a smaller nonsignificant effect on PEF episode severity. Adjusted ratios of geometric means were similar to the previous analysis (table 4). "Wheeze + atopy" had a weaker effect than asthma diagnosis on all outcomes and was not independently predictive of any outcome.

In the second set of regressions, including "wheeze + BHR" and diagnosis, an asthma diagnosis had the stronger significant effect on LRT episode rate and severity, and percentage of symptomatic days. Both factors independently predicted increased peak flow variability, although "wheeze + BHR" had the greater effect.

\section{Discussion}

\section{Summary of findings, and strengths of the study}

The children in this study represent a dedicated subsample of children who reported either wheeze or recurrent cough in a parent-completed questionnaire as part of a population-based survey of asthma. This study has also been able to describe the respiratory morbidity of these children in terms of objectively defined respiratory symptom and peak flow criteria over 13 months, using each child's median symptom or PEF level over the whole study period as their own internal control. The children were highly trained and supervized in these recordings both in this study and in a previous 1 yr study they had just completed.

As a group these children clearly have frequent episodes of cough, wheeze and breathlessness (median 3.7 epi- sodes $\cdot \mathrm{yr}^{-1}$ ) associated with significant airflow obstruction, as measured by deteriorations in peak flow (median 4.2 episodes $\cdot \mathrm{yr}^{-1}$, median fall $55 \mathrm{~L} \cdot \mathrm{min}^{-1}$ ) which were usually prolonged (median duration 16 days). Surprisingly, the frequency and severity of acute symptom or peak flow episodes in 1989 were not strongly related to the presence of reported wheeze or atopy in 1987, although the combination of wheeze and atopy was associated with more frequent acute and chronic lower respiratory symptoms. A pre-existing primary practitioner diagnosis of asthma was, however, both in single and multiple regressions the strongest predictive factor associated with more frequent and more severe lower respiratory episodes, a greater proportion of days symptomatic and a greater overall peak flow variability.

\section{Assumptions and limitations of the study}

The results of this study apply to children who report wheeze and cough; therefore, subgroup comparisons should not be extrapolated to children who report no symptoms. This particular study involved a high degree of commitment and use of procedures involving some discomfort, which decreased participation rates, although the cohort retained the character of the initial sample. It was not practicable to study asymptomatic children in this way.

The "episodes" in this study were identified by computer analysis of the peak flow and symptom charts: this detected many more episodes than were reported by parents. Severity of a symptom episode has been assessed (as many acute asthma scoring systems do) by summing semiquantitative scores for individual symptoms. In addition, self-recorded PEF has been used as a measure of lung function. Peak flow recordings have many pitfalls in childhood, and falls may sometimes reflect lack of effort due to noncompliance, general malaise or interruption by coughing. This particular group of children was highly trained for 12 months prior to this study, and their recordings and technique during exacerbations were frequently checked. Nonetheless, these assumptions should be taken into account when interpreting data regarding episodes so defined. Eighteen children were taking prophylactic inhaled medication, and three were taking oral theophylline. Neither this treatment nor rescue medication 
a)

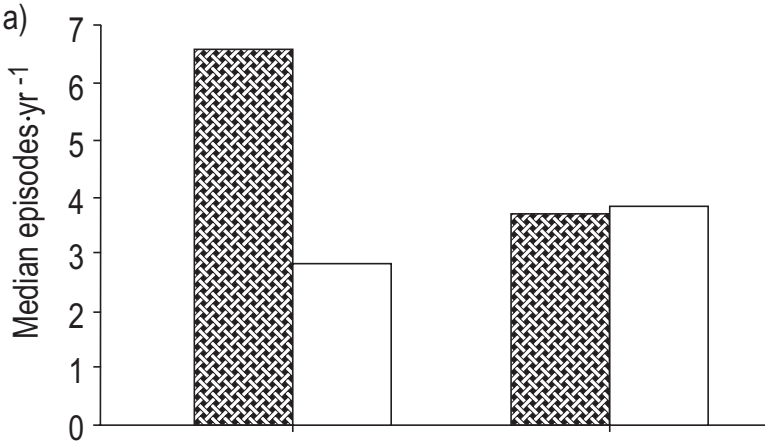

c)

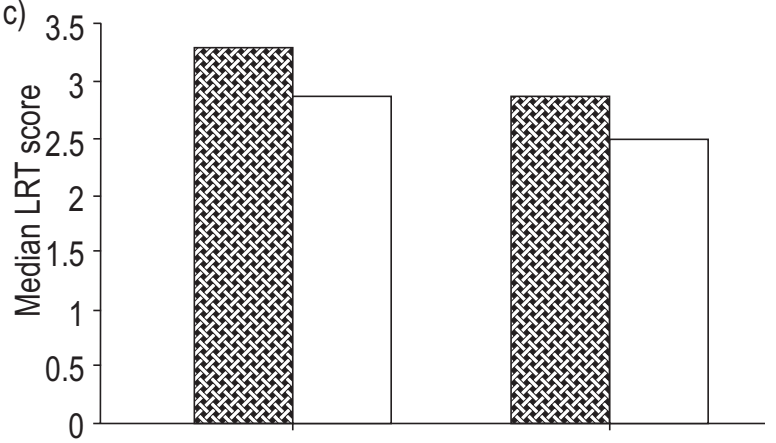

e)

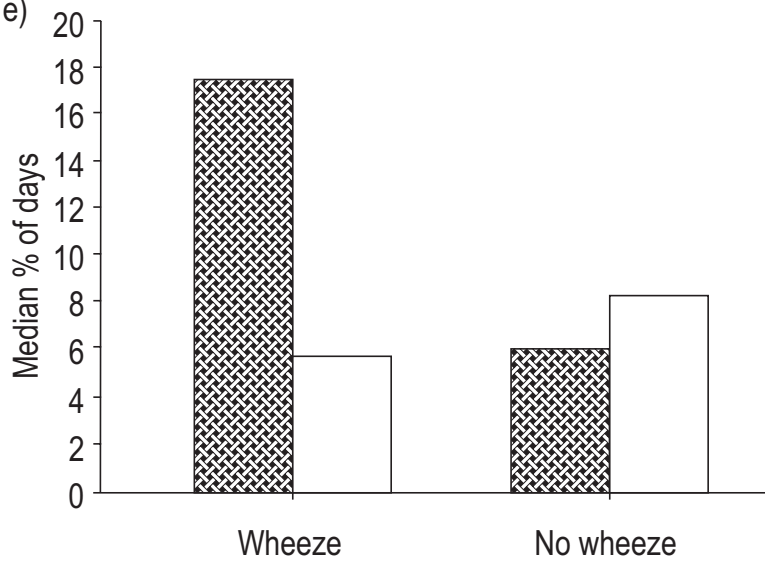

b)

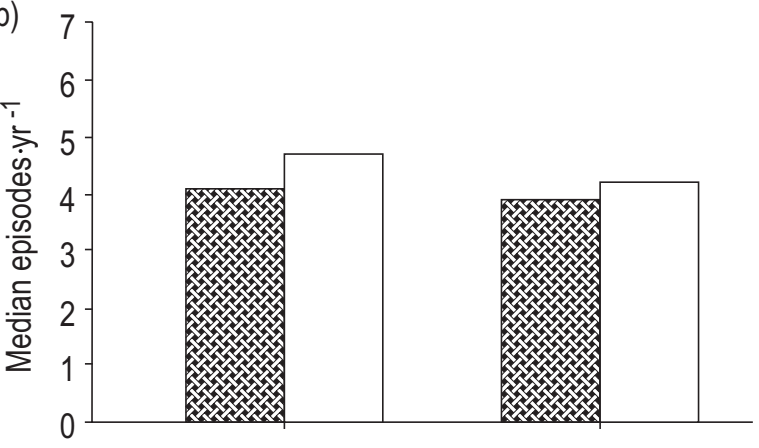

d)

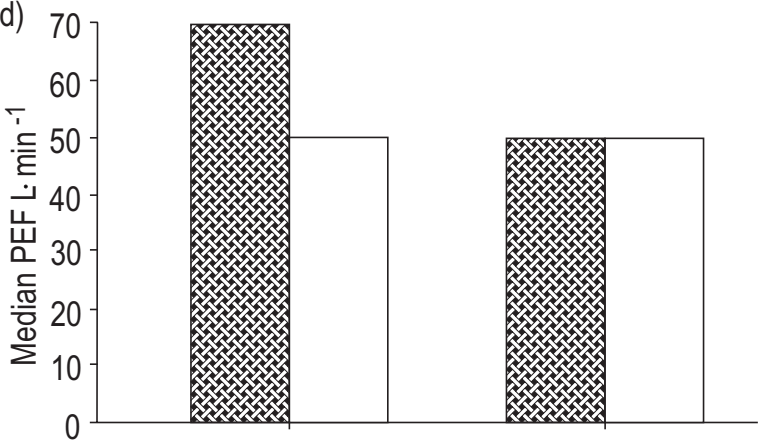

f)

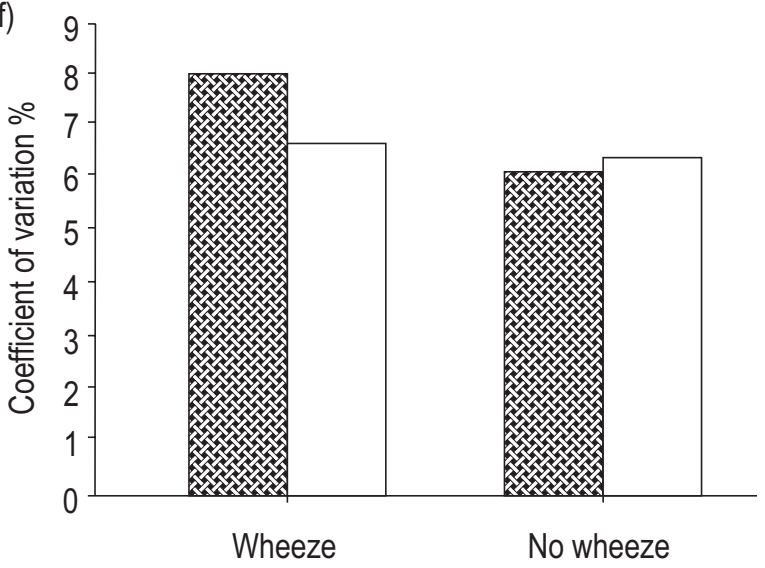

Fig. 2. - Annual rate and severity of lower respiratory tract (LRT) (a and c, respectively) and peak expiratory flow (PEF) episodes (b and d,

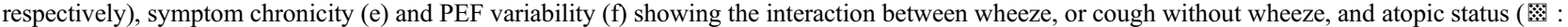
atopic; $\square$ : nonatopic). None of the interactions were significant.

were under control during the study, and the effect of this on episode severity or duration could not be assessed.

\section{Relationship of wheeze versus cough to later respiratory morbidity}

In other studies (see below), wheeze and atopy have been found to be important predictors of respiratory morbidity, particularly "chronic" morbidity rather than morbidity from acute episodes. Clough et al. [4], in the initial study of the current cohort, showed that atopy was associated with lower forced expiratory volume in one second (FEV1), greater peak flow variability and greater "chronicity" of lower respiratory symptoms, defined as the number of symptomatic days as a percentage of the total number of days studied. In that study, the children initially selected for wheeze also had lower FEV1 and greater peak flow variability but had greater "severity" rather than "chronicity" of lower respiratory symptoms after controlling for atopy, ("severity" in this case being defined as the mean lower respiratory symptom score on symptomatic days over the whole year). However, in another study by Clough et al. [12], neither atopy nor wheeze were associated with greater frequency or duration of acute episodes of decreased peak flow, although wheeze was associated with more severe peak flow drops. These results are consistent with the present study in the same children $1 \mathrm{yr}$ later, except that an increased peak flow severity in children with wheeze was not found.

WRIGHT et al. [13] reported that among Tucson children, those who reported both wheeze and recurrent cough at the age of 6 yrs had more respiratory illnesses than those with either symptom alone, who in turn had more illnesses than those with neither wheeze nor cough. KeLLy et al. [14] used parental questionnaires to examine morbidity and personal or environmental factors in Liverpool 
Table 4. - Multiple regression models of episode rates, episode severity and chronicity $(n=106)$

\begin{tabular}{lcccc}
\hline & \multicolumn{4}{c}{ Independent variables } \\
\cline { 2 - 5 } Dependent variable & Wheeze versus cough & $\begin{array}{c}\text { Atopy versus } \\
\text { no atopy }\end{array}$ & $\begin{array}{c}\text { Diagnosed asthma versus } \\
\text { no diagnosis }\end{array}$ & BHR versus no BHR \\
\hline Annual rates & $0.98(0.74-1.31)$ & $1.07(0.81-1.40)$ & $1.71(1.24-2.35)$ & $1.20(0.89-1.63)$ \\
$\quad \begin{array}{l}\text { LRT episodes } \\
\text { PEF episodes }\end{array}$ & $0.95(0.76-1.19)$ & $1.02(0.83-1.26)$ & $1.08(0.85-1.38)$ & $1.11(0.88-1.41)$ \\
Mean severity & $1.13(0.91-1.39)$ & $1.06(0.87-1.29)$ & $1.25(1.00-1.56)$ & $1.02(0.82-1.28)$ \\
LRT episodes & $1.00(0.80-1.26)$ & $0.94(0.76-1.16)$ & $1.21(0.94-1.56)$ & $1.19(0.93-1.51)$ \\
PEF episodes & $1.10(0.73-1.66)$ & $1.13(0.77-1.66)$ & $2.30(1.46-3.64)$ & $1.02(0.66-1.58)$ \\
Chronicity & $1.01(0.82-1.24)$ & $0.85(0.70-1.03)$ & $1.30(1.17-1.81)$ & $1.46(1.17-1.81)$ \\
Days symptomatic \% & PEF coefficient of variation \% &
\end{tabular}

All values are ratios of geometric means (95\% confidence intervals (CI)), adjusted using all four independent variables in the model. BHR: bronchial hyperresponsiveness; LRT: lower respiratory tract; PEF: peak expiratory flow.

children. Compared to children with cough alone, they found a much greater frequency of school absenteeism $\left(>6\right.$ days $\cdot \mathrm{yr}^{-1}$ ) and hospital admission in the children with a combination of cough, wheeze and breathlessness. The authors are not aware of any other studies comparing children in these categories using prospective daily longitudinal data in the way that Clough $[4,9,12]$ and the present study have done.

\section{Explanations of the weak discrimination between wheeze and cough}

There are a number of possible explanations for the lack of a strong predictive effect of wheeze on later acute morbidity in this study. Firstly, it is likely that effects which were present were attenuated by low study power and by the use of a cough group rather than an asymptomatic group for comparison. Secondly, the observed changes in children's symptom patterns over the time period between selection and this study may have weakened the differences $(30 \%$ of those with wheeze at first seemed to stop wheezing, whereas $30 \%$ of those with cough and no wheeze later reported wheeze). Thirdly, chronic symptoms and peak flow variability may be more distinctive features of children with asthma than acute exacerbations.

Each of the above explanations may have some validity, but collectively they do not satisfactorily explain the fact that a pre-existing diagnosis of asthma, subject to the same potential weakening factors, retained a very strong predictive effect for LRT episodes, chronic LRT symptoms and PEF variability after 2 yrs.

If the lack of important discrimination afforded by wheeze versus cough in this study is real, does this mean that cough is as good as wheeze at identifying children with respiratory episodes resembling asthma? Or does it mean that questionnaire-elicited wheeze on its own is little better than cough at identifying such children? The latter interpretation is most consistent with the fact that a preexisting asthma diagnosis did have a strong predictive effect in this group of children. In other words, there was a more symptomatic subgroup and it was much more clearly identified by an asthma diagnosis than by the reporting of wheeze in the original questionnaire. Therefore, as PowELL and PRIMHAK [15] have previously argued, it may be inappropriate for questionnaire surveys of asthma to use "current wheeze", without further elaboration, as a surrogate of important asthma. The situation is somewhat different in clinical practice, where the presence of respiratory symptoms is elicited by interview in the context of a full history and examination.

\section{Controversy about the existence of "cough-variant asth- $m a^{\prime \prime}$}

Cough was described as a presenting feature of asthma in the 1970s [16-18] and this became widely accepted

Table 5. - Multiple regression models adjusting for a diagnosis of asthma together with the interaction term "wheeze + atopy" (model A) and the interaction term "wheeze + bronchial hyperresponsiveness (BHR)" (model B)

\begin{tabular}{|c|c|c|c|c|c|c|c|c|}
\hline & \multicolumn{8}{|c|}{ Independent variables } \\
\hline & \multicolumn{4}{|c|}{ Model A } & \multicolumn{4}{|c|}{ Model B } \\
\hline & \multicolumn{2}{|c|}{ Wheeze + atopy } & \multicolumn{2}{|c|}{ Asthma diagnosis } & \multicolumn{2}{|c|}{ Wheeze + BHR } & \multicolumn{2}{|c|}{ Asthma diagnosis } \\
\hline & $\begin{array}{l}\text { Ratio of GM } \\
\quad(95 \% \mathrm{CI})\end{array}$ & $\mathrm{p}$-value & $\begin{array}{l}\text { Ratio of GM } \\
\quad(95 \% \mathrm{CI})\end{array}$ & $\mathrm{p}$-value & $\begin{array}{l}\text { Ratio of GM } \\
\quad(95 \% \mathrm{CI})\end{array}$ & p-value & $\begin{array}{l}\text { Ratio of GM } \\
\quad(95 \% \mathrm{CI})\end{array}$ & p-value \\
\hline LRT epi & $.21(0.87-1.69)$ & 0.246 & $1.75(1.32-2.33)$ & $<0.001$ & $1.36(0.98-1.88)$ & 0.062 & $1.63(1.21-2.19)$ & 0.002 \\
\hline LRT episode severity & $2(0.80-1.29)$ & 0.902 & $32(1.07-1.63)$ & 0.009 & $1.08(0.86-1.38)$ & 0.501 & $1.27(1.02-1$. & 0.030 \\
\hline$\%$ of days symptomatic & $1.40(0.88-2.23)$ & 0.157 & $2.21(1.47-3.32)$ & $<0.001$ & $1.35(0.85-2.15)$ & 0.208 & $2.17(1.41-3.33)$ & 0.001 \\
\hline PEF episode rate & $0.95(0.73-1.50)$ & 0.671 & $1.15(0.92-1.43)$ & 0.222 & $1.03(0.80-1.33)$ & 0.809 & $1.11(0.88-1.41)$ & 0.362 \\
\hline PEF episode severity & $1.15(0.89-1.50)$ & 0.285 & $1.23(0.98-1.55)$ & 0.074 & $1.22(0.94-1.58)$ & 0.133 & $1.18(0.93-1.49)$ & 0.174 \\
\hline $\begin{array}{l}\text { PEF coefficient of } \\
\text { variation } \%\end{array}$ & $1.07(0.84-1.38)$ & 0.570 & $1.45(1.16-1.80)$ & 0.001 & $1.45(1.14-1.85)$ & 0.003 & $1.25(1.00-1.56)$ & 0.047 \\
\hline
\end{tabular}

Data are presented as adjusted ratios of geometric means (GM) (95\% confidence interval (CI)). Diagnosis $n=42$; wheeze + atopy $n=25$ (overlap with diagnosis $n=19$ ); wheeze + BHR $n=30$ (overlap with diagnosis $n=24$ ). LRT: lower respiratory tract. 
after 1981 [19-21]. These studies may have led to a much more liberal application of the label of asthma to children with cough in the current era of asthma awareness [22]. Some studies have found, as this study did, that some children (although only a minority) presenting with chronic cough subsequently have wheezing $[16,20,21$, 23, 24]. Powell and PrimhaK [15] reported a questionnaire study of 8-9-yr-old children in 1991 and 19931994. Of those reporting nocturnal cough in 1991, only a minority $(20.1 \%)$ reported wheeze in the later questionnaire but this minority had considerable morbidity. In another study [25], 25\% of 6-yr-old children with chronic cough developed wheeze 2 yrs later. This study was unable to study asymptomatic control subjects in a similar follow-up fashion to the study subjects, but WRIGHT et al. [13], using repeated cross-sectional measures, found that wheeze was reported at the age of 11 yrs in $39.8 \%$ of those with only wheeze at 6 yrs, $27.3 \%$ in those with only recurrent cough and $13.6 \%$ in those with neither symptom. The new incidence of diagnosed asthma in 6-11-yrold children followed a similar gradient with lower rates.

Recently there has been concern about overdiagnosis of asthma based on chronic cough alone whereas other common causes such as post-viral cough, pertussis and habit or psychogenic cough have been overlooked [26, 27], a concern that was in fact expressed in several of the original papers about cough described above. The authors of the present study fully agree with this concern, and this study in no way contradicts it. However, this study and the studies cited above support the contention that there are a small number of children who report only recurrent cough in a questionnaire and who go on to develop wheeze and significant morbidity from acute respiratory episodes, most likely asthma. In these children it is possible that recurrent nocturnal coughing was a prodromal feature of asthma. Alternatively, in some children, coughing might be totally unrelated to the later onset of asthma. A third possibility is that some children who report only cough also wheeze but do not recognize or report it. In some of the current children with cough, the pattern of acute respiratory problems was very similar to patterns observed in children reporting wheeze. Figure 3 shows examples of recordings over the 13 months of a child with initial wheeze and a child with initial cough (with no recent wheeze episode reported), demonstrating similar episodes of symptoms and peak flow drops, consistent with episodic airway obstruction.

\section{Pre-existing diagnosis of asthma as a predictor of res- piratory morbidity}

The finding that diagnosed children had more frequent and severe respiratory symptoms than children without a diagnosis is not unexpected, and these characteristics may have been important factors in leading to a diagnosis. What was surprising was the strength and robustness of the effect.

The comparisons show that a doctor's diagnosis of asthma, by whatever means, is picking out something about these symptomatic children that is independent of wheeze, atopy or BHR. As a result, it is able to predict ongoing acute and chronic respiratory symptoms better than any or all of these factors. In other words, as BuRR [28] has previously stated, whereas a general practi- tioner's diagnosis of asthma may have variable or low sensitivity for detecting asthma in the community, it is nonetheless a powerful marker of clinically important asthma. This is most likely because diagnosis takes more account of the clinical context than do survey questions or isolated test results, and because it also involves an intuitive component which is hard to measure or analyse.

There has been a reluctance to use asthma diagnosis as an index or qualifier of asthma prevalence in surveys because of the underdiagnosis substantiated some years ago in the UK and elsewhere [3, 29] and the likelihood of regional variation in diagnostic habit. These difficulties cannot be commented on, as only symptomatic children and these in only one region of the UK were studied. However, these results suggest that perhaps asthma diagnosis should be considered and examined again as a marker of important asthma with which to compare populations of similar background, and make up, and with a similar medical system, for instance different populations within the UK. It should be remembered that in the present study, diagnosis was ascertained by a search of primary care records and not from the survey questionnaire. Whether questionnaire-reported asthma diagnosis is as powerful a discriminator needs further examination.
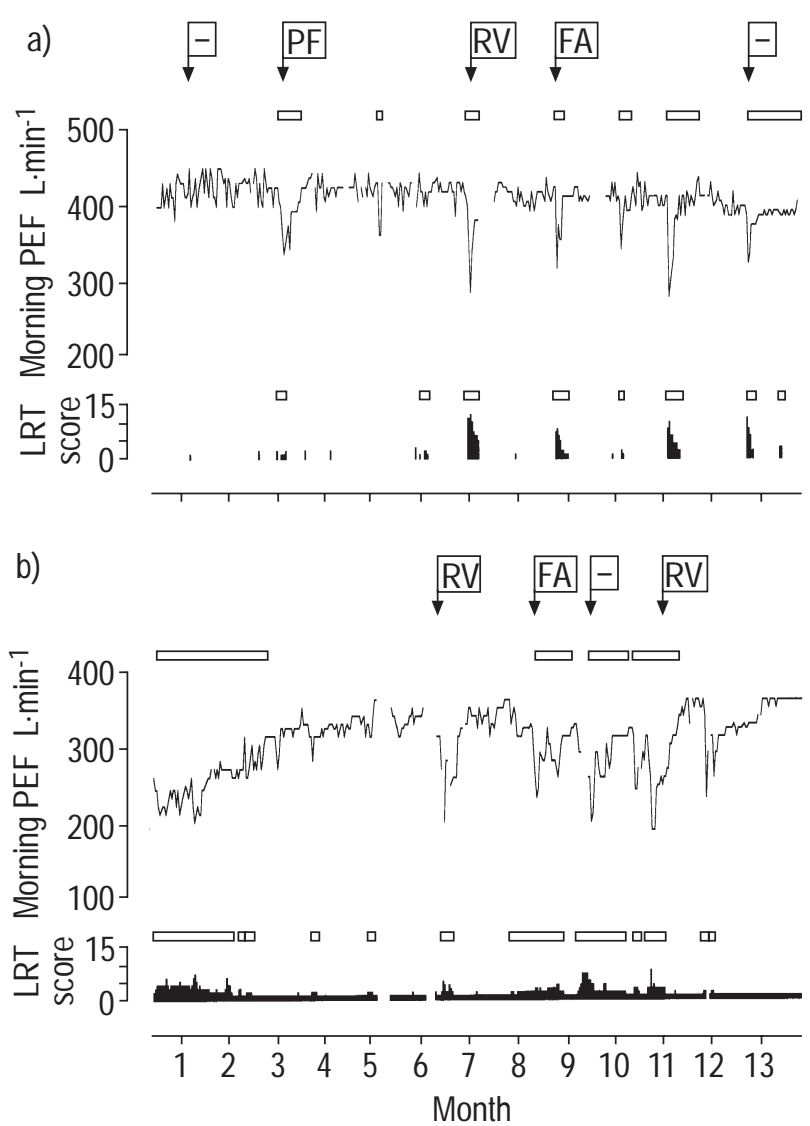

Fig. 3. - Peak flow and symptom score records for: a) a child with initial wheeze and b) a child with initial cough and no wheeze episodes during this study (April 1989-April 1990). Arrows indicate when a report was made and viral samples taken, with letters in boxes indicating viruses identified (PF: parainfluenza; RV: rhinovirus; FA: influenza A; $-=$ no pathogen identified). Computer-defined episodes and their duration are shown as hollow bars above the recordings. LRT: lower respiratory tract; PEF: peak expiratory flow. 


\section{Conclusions}

This study raises questions about the validity of using questionnaire-reported wheeze, without further qualification, as a marker of clinically important asthma in surveys. Assuming that only symptomatic children should be included in prevalence data, it may be more appropriate to enquire both for wheeze and for recurrent cough, and then use another reliable marker to select those with clinically important asthma from both these groups. This study suggests that, in this context, a doctor's diagnosis of asthma is a better marker of ongoing respiratory illness than survey-reported wheeze, atopy or bronchial hyperresponsiveness. Further study and consideration of its usefulness and reliability as a qualifier of reported symptoms in asthma surveys is warranted.

\begin{abstract}
Acknowledgements. The authors thank all the children and their parents who contributed to this study, and I. Asher, A. Leversha, D. Teele, G. Marks and A. Chang for their helpful comments on the manuscript.
\end{abstract}

\section{References}

1. Asher MI, Keil U, Anderson HR, et al. International Study of Asthma and Allergies in Childhood (ISAAC): rationale and methods. Eur Respir $J$ 1995; 8: 483-491.

2. The International Study of Asthma and Allergies in Childhood (ISAAC) Steering Committee. Worldwide variation in prevalence of symptoms of asthma, allergic rhinoconjunctivitis, and atopic eczema ISAAC. Lancet 1998; 351: 1225-1232.

3. Speight ANP, Lee DA, Hey EN. Underdiagnosis and undertreatment of asthma in childhood. BMJ 1983; 286 1253-1256.

4. Clough JB, Williams JD, Holgate ST. Effect of atopy on the natural history of symptoms, peak expiratory flow, and bronchial responsiveness in 7- and 8-year-old children with cough and wheeze: a 12-month longitudinal study. Am Rev Respir Dis 1991; 143: 755-760.

5. Peat J, Toelle B, Salome C, Woolcock A. Variability of bronchial responsiveness and respiratory symptoms in a cohort of Sydney schoolchildren studied over one year. Eur Respir J 1993; 6: 662-669.

6. Pattemore PK, Holgate ST. Bronchial hyperresponsiveness and its relationship to asthma in childhood. Clin Exp Allergy 1993; 23: 886-900.

7. Johnston SL, Sanderson G, Pattemore PK, et al. The polymerase chain reaction in the diagnosis of picornavirus infection in subjects with and without respiratory symptoms. J Clin Microbiol 1993; 31: 111-117.

8. Johnston SL, Pattemore PK, Sanderson G, et al. Community study of role of viral infections in exacerbations of asthma in 9-11 year old children. BMJ 1995; 310: 12251229.

9. Clough JB, Williams JD, Holgate ST. Profile of bronchial responsiveness in children with respiratory symptoms. Arch Dis Child 1992; 67: 574-579.

10. Yan K, Salome C, Woolcock A. Rapid method for measurement of bronchial responsiveness. Thorax 1983; 38: $760-765$.

11. Johnston SL, Pattemore PK, Sanderson G, et al. The relationship between upper respiratory infections and hospital admissions for asthma: a time-trend analysis. $\mathrm{Am}$ J Respir Crit Care Med 1996; 154: 654-660.

12. Clough JB, Holgate ST. Episodes of respiratory morbidity in children with cough and wheeze. Am J Respir Crit Care Med 1994; 150: 48-53.

13. Wright AL, Holberg CJ, Morgan WJ, Taussig LM, Halonen M, Martinez FD. Recurrent cough in childhood and its relation to asthma. Am J Respir Crit Care Med 1996; 153: 1259-1265.

14. Kelly YJ, Brabin BJ, Milligan PJM, Reid JA, Heaf D. Clinical significance of cough and wheeze in the diagnosis of asthma. Arch Dis Child 1996; 75: 489-493.

15. Powell CVE, Primhak RA. Stability of respiratory symptoms in unlabelled wheezy illness and nocturnal cough. Arch Dis Child 1996; 75: 385-391.

16. Corrao WM, Braman SS, Irwin RS. Chronic cough as the sole presenting manifestation of bronchial asthma. $N$ Engl $J$ Med 1979; 300: 633-637.

17. McFadden ER Jr. Exertional dyspnoea and cough as preludes to acute attacks of bronchial asthma. $N$ Engl $J$ Med 1975; 292: 555-559.

18. Mellis CM, Levison $\mathrm{H}$. Chronic cough and bronchial reactivity in children. Am Rev Resp Dis 1977; 115: 286-.

19. Taussig LM, Smith SM, Blumenfeld R. Chronic bronchitis in childhood: what is it? Pediatrics 1981; 67: 1-5.

20. Cloutier MM, Loughlin GM. Chronic cough in children: a manifestation of airway hyperreactivity. Pediatrics 1981; 67: 6-12.

21. König P. Hidden asthma in childhood. Am J Dis Child 1981; 135: 1053-1055.

22. Silverman M, Wilson N. Asthma - time for a change of name? Arch Dis Child 1997; 77: 62-64.discussion 64-65.

23. Hannaway PJ, Hopper GD. Cough variant asthma in children. JAMA 1982; 247: 206-208.

24. Parks DP, Ahrens RC, Humphries CT, Weinberger MM. Chronic cough in childhood: approach to diagnosis and treatment. J Pediatr 1989; 115: 856-862.

25. Lewis HM. Cough - but is it asthma? [letter; comment]. Arch Dis Child 1994; 70: 554.

26. McKenzie S. Cough - but is it asthma? [annotation]. Arch Dis Child 1994; 70: 1-2.

27. Chang AB, Phelan PD, Carlin JB, Sawyer SM, Robertson CF. A randomised, placebo controlled trial of inhaled salbutamol and beclomethasone for recurrent cough. Arch Dis Child 1998, 79: 6-11.

28. Burr ML. Diagnosing asthma by questionnaire in epidemiological surveys [editorial]. Clinical \& Experimental Allergy 1992; 22: 509-510.

29. Anderson HR, Bailey PA, Cooper JS, Palmer JC, West S. Medical care of asthma and wheezing illness in children: a community survey. J Epidemiol Commun Health 1983; 37: $180-186$. 